\title{
Putting Dementia into Context
}

\section{A Selective Literature Review of Assistive Applications for Users with Dementia and Their Caregivers}

\author{
Joël Vogt ${ }^{1}$, Kris Luyten ${ }^{2}$, Jan Van den Bergh ${ }^{2}$, \\ Karin Coninx ${ }^{2}$, and Andreas Meier ${ }^{1}$ \\ 1 Department of Informatics, University of Fribourg, Boulevard de Pérolles 90, \\ CH-1700 Fribourg, Switzerland \\ \{joel.vogt, andreas.meier\}@unifr.ch \\ 2 Hasselt University - tUL - IBBT, Expertise Centre for Digital Media, \\ Wetenschapspark 2, B-3590 Diepenbeek, Belgium \\ $\{$ kris.luyten, jan. vandenbergh, karin. coninx @uhasselt. be
}

\begin{abstract}
People with dementia face a decline of their cognitive functions, including memory impairment and difficulty to orient in time and space. Assistive applications can ease the effects of dementia by assuming and supporting impaired functions. Context-awareness is an accepted paradigm for assistive applications. It enables interactive systems to react appropriately to situations that occur during daily routines of people with dementia. However, there currently is no recommended framework to view symptoms of dementia in terms of context and context-awareness. The aim of this paper is to inform designers in the early design stages of assistive applications how requirements and needs of people with dementia can be represented in a context-aware application. Based on a systematic literature review, we elicit which context types are linked to the needs of people with dementia and their caregivers and how they are used in existing assistive applications in dementia care. Our focus is on applications evaluated and assessed with people with dementia. We also classify these assistive applications by the offered context-aware services. We observe that these should not be limited within the realm of the local residence; context types that are valuable in-house can, to a certain extent, also be leveraged outside a local residence. We believe the proposed framework is a tool for application builders and interface designers to accomplish an informed design of systems for people with dementia.
\end{abstract}

Keywords: Dementia, Independent living, context-awareness, assistive applications.

\section{Introduction}

Dementia affects about $4 \%$ of people over the age of 65 and $40 \%$ of people over 90. In Europe, at least half of the elderly population affected by dementia, have a mild form of dementia 33. The organization Alzheimer's Disease International 
estimates that the worldwide cost of dementia amounts to $1 \%$ of the global gross domestic product, or USD 604 billion [50].

Literature shows that the dementia syndrome itself does not thwart the use of digital assistive application, at least at its early stages [233045]. To what extent People with Dementia (PwDs) can benefit from assistive applications greatly depends on the ability to accommodate the user's cognitive impairments and other needs 23/313845]. This limits the use of standardized ICT applications [40 23]. Moreover, assistive applications should be appealing to their users and not be perceived as stigmatizing [3145]. Hughes et al. 21] emphasize the importance to respect the dignity of PwDs. Context-aware computing allows the development of interactive systems that are aware of their users and behave accordingly 9111551. The context-aware paradigm considers the users and the operating environment in the application design and behavior. Given the number of people with dementia and their desire for solutions to cope with dementia, building interactive systems tailored for this user group is extremely important.

\subsection{Aims of the Paper}

The aim of this paper is to provide a reference framework that assists the preliminary phase in the design and development of assistive applications for PwDs. Given the difficulties to evaluate this type of applications with the target audience, it is vital to maximize an informed design approach 338. We approached this by analyzing services of context-aware assistive applications in dementia care and how they exploit context. We also study the type of context that these applications use to address their users' needs.

\subsection{Approach}

We conducted a systematic literature review of projects that developed assistive applications for PwDs. The design and development of assistive applications for PwDs is a complex and error-prone task. While traditional user-centered techniques are suitable for abled-body people, Newell et al. 34. point out that they provide little guidance as to how to work with users with special needs. Traditional user-centered design encourages members of the design team to make their design choices from the user's perspective. However people with severe cognitive impairment are too diverse and their situation cannot be reflected as an average user. In addition a user group of people with disabilities has a greater variety of characteristics of functionality which may also be conflicting [34]. We therefore focused on projects which evaluated their running applications with PwDs. We further limited the scope to applications that react to context during runtime (in contrast to design time adaption to contexts of use). Additionally, we considered only literature we could access from our university network or library.

In a first step, we searched the bibliographical databases of ACM, IEEE, ScienceDirect and PubMed. The search query specified the following criteria for the abstract or title of literature: (dementia OR alzheimer) AND (context 
OR location OR activity OR ambient OR situation) AND (person OR people OR individual OR adult). ACM gave 10 results, IEEE 34, ScienceDirect 5 , PubMed 1 . In a second iteration, we removed candidates that did not evaluate and assess their technical solutions with PwDs. This clearly showed that very few of the research projects actually included user trials: ACM was left with 1 result [5], IEEE 0, ScienceDirect 1 [44, PubMed 1 [45]. The number of publications on dementia and context that also performed evaluations with end-users are very low and does not provide sufficient material to define a design space for creating assistive applications. However, we used these papers as a starting point and elaborated on the respective projects they originated from. In a third iteration, we therefore searched references for projects that focused on assistive applications in dementia care. This led to 3 additional Research and development projects 12 24 32].

\section{The Person with Dementia as the User}

This section puts a human face on dementia. It discusses the impact of dementia, in particular Alzheimer's disease (AD), on People with Dementia (PwDs) and their social context society in literature, to gain a sense of the complexity of this disease.

\subsection{The Dementia Syndrome}

Dementia is a syndrome of serious decline of cognitive abilities that exceed agerelated decline. Common symptoms are memory problems, difficulties to perform familiar tasks, impaired judgement, language deterioration, and mood changes. Dementia is a syndrome with different etiologies, of which $\mathrm{AD}$ is the most common type [227]. Forgetfulness is generally seen as the primary symptom of dementia; however, this is not necessarily the case for every pathology. Usually, cognitive abilities irreversibly deteriorate over time and the underlying disease is incurable. There is not a single definition of dementia. However, they all do require a functional disability threshold that has to be crossed. Individuals can therefore exhibit a decline of cognitive functionalities that do meet the criteria for dementia [27. Mandell and Green [27] describe dementia as "a syndrome of acquired persistent intellectual impairments characterized by deterioration in at least three of the following domains: memory, language, visuospatial skills, personality or behavior, and manipulation of acquired knowledge (including executive function)".

\subsection{Alzheimer's Disease}

$\mathrm{AD}$ is a neurodegenerative disease and the most common form of dementia [2]. Currently $\mathrm{AD}$ can neither be slowed down nor cured [2]. Due to the complexity of $\mathrm{AD}$, some even doubt whether a cure can ever be found [36]. AD is commonly diagnosed after the age of 65 , although brain damage resulting from $\mathrm{AD}$ can 
start 10 to 20 years before first symptoms appear. The cause of AD is unknown. Memory problems that exceed age-related decline are early indicators. when the damage spreads to hippocampus. As AD advances, other impairments in other cognitive domains become apparent that curtails abilities to perform everyday tasks, language difficulties, difficulties to understand visual images and spatial relationships, confusion over time and space, misplacing and losing items, mood changes and social isolation [27.

Hodges [17] characterize the clinical process of AD as stages of patterns of impairment of the cognitive abilities memory, attention and executive abilities, language, visuospatial and perceptual and praxis. The first stage, mild cognitive impairment, is prior to the dementia onset. The worsening of cognitive abilities is characterized by the stages mild dementia, moderate dementia and severe dementia. While early on in the clinical process deterioration of abilities vary from individual to individual, the later developments follow similar patterns. However the duration of each stage differs from person to person.

\subsection{Needs of People with Dementia}

Dementia is often associated with forgetfulness and confusion. Temporal and memory impairment mean that PwDs need to be reminded of future and past activities and events 1316. Memory impairment also manifests itself as an increasing need for information about everyday issues. Hawkey et al. [16] cite an interviewee who wanted to know the role of the participating person (in this example a physician), the location of this person and the route, departure time, and what to wear. The authors also found that some participants had difficulties to relate with time or recognize people they know.

A cause for frustration was the increasing difficulty of some participants to use familiar devices and initiating or completing even simple tasks [13 16 49]. Wherton and Monk [49] analyzed the difficulties $\mathrm{PwDs}$ are faced with when performing Activities of Daily Living (ADL) and how they can be assisted. They found that the difficulties were resulting from sequencing problems, problems finding things, problems operating appliances and incoherence. Sequencing problems occur when incorrect objects or actions for a given step are used. This was seen as a result of being unable to control stimulation from objects or affordances that are not relevant for that task. The second cause for this problem is the degeneration of semantic memory. The impairment makes it harder for the Person with Dementia $(\mathrm{PwD})$ to distinguish similar objects types. Episodic memory problems led to forgetting steps that were already taken; hence, repeating them. Omissions of sub-goals were also reported, when the sub-goal was too similar to the overall goal. The problem finding items was seen for one as a result of episodic memory loss. The PwD would not remember which places were already searched and repeat the search. The disability to identify visible items was also attributed to either not being able to subdue task-irrelevant stimulation or semantic memory impairment. The problem with using items was seen attributable to the inability 
to comprehend the use of an appliance. The participants did not know when to use an appliance or found it too difficult to understand its functionality. This is seen as a result of not being able to select between action schemas or the inability to remember the actions that need to be performed. Incoherence is seen as the problem when PwD engaged in activities that are not related to the overall goal.

Dementia aid is primarily situated within the residence of the PwDs, not on activities outdoors. Even though PwDs, at least at the early stages of their illness, appreciate outdoor activities [4. However caregivers (CGs) fear for their love one's safety and are therefore reluctant to let their PwD leave their safe environment alone. PwDs may also be anxious about getting lost because they are uncertain about their location and finding their way home 413. PwDs feel more conformable in familiar places. Brittain et al. 4. found that PwDs can use landmarks or ask other people to find their bearings. One interviewee would use photographs of older buildings to link the past memory with the current landscape. To reduce uncertainty about the PwD location, Wherton and Monk [4] suggest to reinsure both parties by mutually sharing contextual information. Through mutual awareness, "the function of the technology becomes communication instead of monitoring" 47.

Social interaction is vital for PwDs, e.g. 4133147]. However, the increased difficulty to manipulate items and problems impairment rises the bar for the PwD to interact with their social environment. Being more and more confined to their residence also affects social activities 4 .

\subsection{Challenges When Developing for Users with Dementia}

Involving $\mathrm{PwDs}$ and their CGs in the design process requires empathy for the users and awareness of their abilities, needs and to their environment [1 31 38. CGs hold considerable sway over the PwD and need to be convinced of the project's merits [3]. PwDs are in most cases older people who are affected by age related degeneration of physical abilities such as vision, hearing or tremor [16. PwDs may be more or less aware of their conditions [16. PwDs are also prone to emotional changes. They are easily irritated and may be insecure about their contribution or even be frustrated or anxious 28,38.

It is therefore difficult for designers who have no previous contact with $\mathrm{PwD}$ to grasp the complexity of such users. Yet involving $\mathrm{PwDs}$ is imperative to gain an understanding of their very unique needs and requirements 63846. As Stalker et al. 46] point out, if the $\mathrm{PwD}$ is the user, then "Reliance on carers for this information is foolhardy".

Novelty is often seen as problem for PwDs. Orpwood et al. 38, argue that "People with dementia will not be able to learn to use new devices in their home". Wherton et al. 48, offer a more nuanced perspective, suggesting that novelty itself is not the problem but rather "learning new arbitrary mappings and procedures". They found that direct implicit clues worked well to guide the PwD. 


\section{Context and Context-Awareness}

Newell et al. 35 suggest that user interface design for people with cognitive impairment should mitigate memory impairment, avoid cognitive overload and take into account individual characteristics. While to a certain degree these requirements are desired by all users, the reduced cognitive abilities of People with Dementia ( $\mathrm{PwDs}$ ) and the emotionally very demanding situation makes these rules imperative. Context-awareness is an accepted paradigm to adapt assistive applications to known contexts of use during design time and can adapt to a dynamic environment during runtime [15/51.

This section presents the categories of context variables and context-aware behaviors for dementia care that were identified in literature. We use the definition of context and context-awareness as provided by Dey and Abowd [9]. Their definition is as follows: "Context is any information that can be used to characterize the situation of an entity. An entity is a person, place, or object that is considered relevant to the interaction between a user and an application, including the user and applications themselves. [... A system is context-aware if it uses context to provide relevant information and/or services to the user, where relevancy depends on the users task.". This implies that context is a fairly broad concept. Their definition does however provide helpful cues to determine if a piece of information is context or not: It puts contextual information within the scope of the interaction between the user and the system and it does not limit context-aware behavior strictly to context that is obtained during runtime.

Dey and Abowd propose four primary context dimensions to describe the situation of a subject:

1. activity: the intrinsic properties that determine the state of an entity. E.g. feelings, tasks or status.

2. identity: the extrinsic properties that describe an entity independent of time and location. For example the name of a person, the phone number or personal relationships.

3. location: the location of an entity in a physical space.

4. time: the location of an entity in temporal space.

Section 3.1 extends Dey and Abowd's categorization of context for assistive applications in dementia care. We studied the research and development projects identified in Sect. 1.2 and literature on the needs and requirements of discussed in Sect. 2.3 to elicit the second level context types that are relevant to provide services and information to PwDs and their caregivers (CGs). Literature on needs and requirements drew our interest to identify context types that have not yet been used in current applications. We also studied the runtime behavior of assistive applications for $\mathrm{PwDs}$ in literature that use context to provide relevant services and information to the user. Section 3.2 summarizes our findings as context-aware services. 


\subsection{Context Types for Dementia Care}

This section discusses the context types that our literature review found represent dementia related deficits for assistive applications.

Activity. The activity context type describes the intrinsic properties of an entity [. Dementia affects the ability of PwDs to perform activities and influences their emotions. Their abilities and emotional state influence the dialog between a Person with Dementia ( $\mathrm{PwD}$ ) and the assistive application. Activity also describes the capabilities and state of devices. The following context types were found:

- Emotion: Dementia influences a person's emotions and their ability to control emotions [4] and anxiety [1644. Rebentisch et al. 43] reckon that frustration/fear, confusion and anger were influential emotions that indicate the onset inappropriate behavior. Hoey et al. 18] consider the variables dementia_level, awareness and responsiveness for their activity assistant.

- Capabilities: Capabilities describe services or functionality of an entity. PwDs expressed the desire to know more about whom they are interacting with [16], distinguish between similar items [49] or how to use a device [16 49]. This value helps to define semantics of entities, including competencies of people, types of places, and device functionalities.

- Action: The action describes the goal oriented process an entity is performing 6]20]41].

- Status: The status describes the current state of an entity. For people, this context type can describe their availability [26]. For household appliances, the status can describe whether they are on or off [6].

- Preferences: Assistive applications should consider the users' personal preferences [16]. This context type specifies stable personal preferences, meaning they are not subject to frequent change. Examples include language settings, date formats, or color settings; e.g. [6].

Identity. Identity is needed to identify participants and their relationships. The name of an entity and Social relationship is of particular importance in dementia care. The social relationship context type links two or more persons. PwDs are increasingly dependent on their social context for care and social activities, e.g. [4]13[16]47]. Furthermore, PwDs may experience difficulty to recognize how they are related to some people in their social context 1647.

Location. PwDs experience difficulty to orient themselves physical space, even in once familiar places 4|16. They may not recognize their current location or remember how to reach a certain destination, for example their home. We identified the subtypes: orientation, position, relative location and semantic location. Orientation shows the direction in which to move from one place to another. The position determines the current location, for example for navigation assistance or monitoring. A semantic location denotes the meaning of a place. For example a "bakery" is a place that has a bread service. Hence, if the PwD's semantic location is "bakery", this person is in that place. 
Time. Dementia affects episodic memory and temporal orientation 16 47. PwDs find it increasingly difficult to recall recent events, plan activities and how to relate to time. Precise time, time span and semantic time were identified. Precise time and time span are used to set reminders, either precise or within a certain threshold. Semantic time refers to an event entity in the user's context, for example lunch time or the time to watch the evening news.

\subsection{Categories of Context-Aware Services}

The following services were identified in our literature review:

Reminder. A reminder system is a memory assistant to plan and structure future events and activities. The system triggers reminders when certain conditions are met (e.g. time or location is reached). A notable example of context-aware reminders is the CybreMinder [10. In dementia care, HYCARE [14] supports setting reminders with several context types.

Activity Assistant. While reminder systems remind their users when activities are due, activity assistance tells users how to perform Activities of Daily Living (ADL). Activity assistants vary in activities they support, and the type of support they provide. The COGKNOW DayNavigator [6] displays video sequences that show how to perform a task. The user interacts with a touch screen to play a sequence. Automatic activity assistants delve in the realm of artificial intelligence. The user's context is monitored to intervene when the user is deviating from the plan to complete the task (e.g. 20/4144]). The development of intelligent activity assistants still faces many challenges [19]. The $\mathrm{COACH}$ system is seen as the most sophisticated ADL assistant [19. It guides a PwD through the hand washing process [20].

Information Provider. Information providers use context to present relevant functions or information objects to the user. The COGKNOW DayNavigator is an example for the former. It offers simplified access to in-house devices and other services. The functions available change when the $\mathrm{PwD}$ is outdoor.

An example for the latter are 'information appliances' 16. These applications provide PwDs detailed information about previous, ongoing, and future activities. The MemoryLane project is exploring this area in dementia care [22].

Communication and awareness. Communication and awareness systems extend social relationships and awareness beyond time and location. Communication is either asynchronous or synchronous. For example, the COGKNOW DayNavigator has a picture-based interface to a stationary and mobile phone. The awareness function makes one entity's context visible to others. An application of awareness is dementia care is monitoring. For example the KITE system 45] shows the location of the PwD to the CG. A context-aware system for PwDs was proposed by [26]. The PwD uses a mobile phone to communicate with his social network. The system stores the type of social relationship for each contact and their availability. The personal CG can monitor the PwD's current location and schedule. 
Navigation. A navigation system guides a user through physical space to a desired destination. Both the COGKNOW 32] and KITE 45] projects have a guidance function to help the $\mathrm{PwD}$ find their way home. A navigation system that considers location and time was proposed by [26]. Todo items are placed in the PwD's time, location and social context. The navigation system helps the $\mathrm{PwD}$ to find the location of a given todo list item in a timely manner.

Life-Logging. Life-Logging complements a person's memory function. It records a constant stream of information during the day as images, sounds, location and time. The information is later processed to reconstruct the user's experiences 2422].

\section{Research Projects}

This paper considers the context-aware properties as an inherent part of most assistive applications for People with Dementia (PwDs) and their caregivers (CGs) to accommodate the users' needs and requirements in a dynamic environment. The motivation for this paper is to provide designers and developers with a tool to make an informed decision on the use of context and context-awareness. We

Table 1. Categories of context-aware applications in dementia care

\begin{tabular}{|c|c|c|c|c|c|c|c|c|c|}
\hline \multicolumn{4}{|c|}{ Project } & \multicolumn{6}{|c|}{ Category } \\
\hline $\begin{array}{l}\text { Е } \\
\text { Z } \\
Z\end{array}$ & 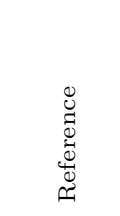 & 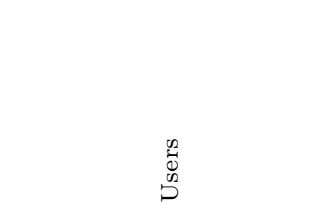 & 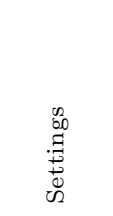 & 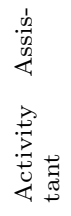 & 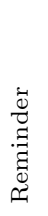 & $\begin{array}{l}\dot{0} \\
00 \\
60 \\
0 \\
.1 \\
\stackrel{1}{0} \\
.13\end{array}$ & 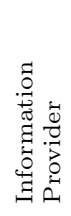 & 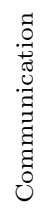 & $\begin{array}{l}2 \\
.0 \\
\pi \\
.0 \\
.0 \\
\frac{\pi}{2} \\
Z\end{array}$ \\
\hline KITE & 45 & $\begin{array}{l}\text { People with mild to mod- } \\
\text { erate dementia, caregivers }\end{array}$ & outdoor & & & & & $x$ & $\times$ \\
\hline $\mathrm{COACH}$ & $18|20| 29$ & $\begin{array}{l}\text { People with moderate-to- } \\
\text { severe dementia }\end{array}$ & indoor & $\times$ & & & & $\times$ & \\
\hline COGKNOW & \begin{tabular}{|l|l|}
6 & 32 \\
\end{tabular} & $\begin{array}{l}\text { People with mild demen- } \\
\text { tia, caregivers }\end{array}$ & $\begin{array}{l}\text { indoor, } \\
\text { outdoor }\end{array}$ & $x$ & $x$ & & $x$ & $x$ & $\times$ \\
\hline $\begin{array}{l}\text { Context- } \\
\text { Aware } \\
\text { Wayfinder }\end{array}$ & 5 & $\begin{array}{l}\text { Mental retardation, } \\
\text { epilepsy, organic depres- } \\
\text { sion, Parkinsonś Disease, } \\
\text { dementia, schizophrenia, } \\
\text { organic brain syndrome }\end{array}$ & $\begin{array}{l}\text { indoor, } \\
\text { outdoor }\end{array}$ & & & & & $\times$ & $\times$ \\
\hline CPVS & 12 & $\begin{array}{l}\text { People without cognitive } \\
\text { impairment, people with } \\
\text { mild dementia, caregivers }\end{array}$ & $\begin{array}{l}\text { indoor, } \\
\text { outdoor }\end{array}$ & & $\times$ & & & $x$ & \\
\hline ExPress Play & 44 & $\begin{array}{l}\text { People with mild to mod- } \\
\text { erate dementia }\end{array}$ & indoor & $\times$ & & & & & \\
\hline MemExerciser & 24 & $\begin{array}{l}\text { People with mild cog- } \\
\text { nitive impairment, mild } \\
\text { Alzheimer,moderate } \\
\text { Alzheimer, caregivers. }\end{array}$ & $\begin{array}{l}\text { indoor, } \\
\text { outdoor }\end{array}$ & & & $x$ & & & \\
\hline
\end{tabular}


Table 2. Context Types in dementia care

\begin{tabular}{|c|c|c|c|c|c|c|c|c|c|c|c|c|c|c|}
\hline \multirow{3}{*}{ Projects } & \multicolumn{14}{|c|}{ Context Type } \\
\hline & \multicolumn{4}{|c|}{ ACtivity } & \multicolumn{3}{|c|}{ IDENTITY } & \multicolumn{4}{|c|}{ LOCATION } & \multicolumn{3}{|c|}{ Time } \\
\hline & 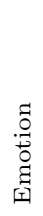 & 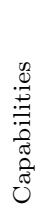 & 苛 & 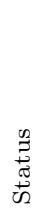 & 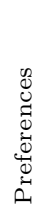 & 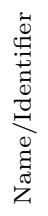 & 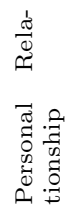 & 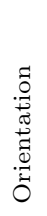 & 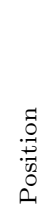 & 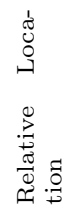 & 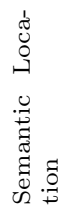 & 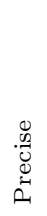 & $\begin{array}{l}\text { त् } \\
\text { थิ } \\
\text { की }\end{array}$ & 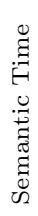 \\
\hline KITE & & & & $\times$ & & $\times$ & $\times$ & $\times$ & $\times$ & & & $\times$ & & \\
\hline $\mathrm{COACH}$ & $\times$ & $\times$ & $\times$ & & & $\times$ & $\times$ & $\times$ & $\times$ & $\times$ & & $\times$ & & \\
\hline COGKNOW & & $\times$ & $\times$ & $\times$ & $\times$ & $\times$ & $\times$ & $\times$ & $\times$ & & & $\times$ & $\times$ & $\times$ \\
\hline $\begin{array}{l}\text { Context-Aware } \\
\text { Wayfinder }\end{array}$ & & & & & & $\times$ & $\times$ & $\times$ & $\times$ & & & $\times$ & & \\
\hline CPVS & & & & $\times$ & $\times$ & $\times$ & $\times$ & & & & & $\times$ & & $\times$ \\
\hline ExPress Play & $\times$ & & & & & & & & & & & $\times$ & & \\
\hline MemExerciser & & & & & & & & $\times$ & $x$ & & & $\times$ & & \\
\hline
\end{tabular}

approach by presenting the assistive applications identified in Sect. 1.2 and discussed in more detail in Sect. 4.1 from a context and context-aware perspective. Table 1 shows who the users were, the types of context-aware services they used (see Sect. 3.2) and where the applications were deployed (i.e. indoors or outdoors). Table 2 shows which context types (see Sect. 3.1) assistive applications relied on to provide those services to their users.

\subsection{Context-Aware Assistive Applications}

The following projects were identified in Sect. 1.2 for our literature review:

KITE. The Keeping In Touch Everyday (KITE) 45] project developed an outdoor navigation and communication system for PwDs. The Person with Dementia $(\mathrm{PwD})$ carries a mobile device. It has a call button to contact the $\mathrm{CG}$ and a navigation function. The navigation function presents the $\mathrm{PwD}$ with instructions to find their way home. Additionally, the mobile device tracks the PwD's position as well as the device and alert state. These contextual information is presented to the CG via a web interface.

$C O A C H$. COACH [182029] is an activity assistant for the hand washing process. The system visually tracks the user's hand movement and process relevant items. The system bases its decisions on current observations and the user's level of dementia and emotions. Emotions are referred to as attitude (dementia_level, awareness and responsiveness [18]). Only when the $\mathrm{PwD}$ experiences difficulties with the task, COACH presents the user with either visual or audio prompts. If prompts are ineffective and the $\mathrm{PwD}$ resume the task, the $\mathrm{CG}$ is called. The user's comportment influences the attitude and dementia variables over time. 
COGKNOW. COGKNOW 671432 is a EU funded project to develop a comprehensive support system for people with mild dementia. COGKNOW extends the the realm of the PwD's residence. The PwD accesses services either through a stationary touchscreen interface or a mobile device. Activities are supported with a context-aware reminder system, video recorded instructions for a set of activities and simplified access to services. The reminder system triggers timebased and event-based reminders. Reminders are set by a CG [14. Simplified access to music, radio and communication service are presented by both stationary and mobile device. Outdoors, the PwD has access to reminders and communication on the mobile device. An additional navigation function to get home is presented. Social communication is provided with a simplified interface to the phone. The $\mathrm{PwD}$ is presented with pictures of each CG to call. A help button is provided to directly call the main CG.

Context-Aware Wayfinder. [5] developed a navigation system for people with cognitive impairments, including dementia. The system guides their users to a destination by presenting images of the next waypoint and overlaying directions for the user's route. User and CG can call if needed. The system records the route progress and compliance. It presents this monitoring information to an authorized person.

ExPress Play. ExPress Play is an application for PwDs to engage in a creative activity through music. The system provides a touch screen and an easy to use interface. Users can select their emotion (happiness, sadness and anger) which influences the music being played and the visualization of the sound. The users interact with the application by dragging a finger across the screen. The system draws shapes depending on the speed of movement and plays different sounds 44.

CPVS. The Cell Phone Video Streaming (CVPS) system [12] is a mobile reminder for people with mild dementia. The system consists of a smart phone for the PwD and a workstation with video camera for the CG. Reminders are video clips that are presented to the PwD. The PwD's CG records and schedules the reminders for the user. The smart phone downloads videos and notifies the PwD at the given time. The PwD needs to confirm the reminder by pressing a button. Reminders' confirmation state is accessible to the CG via a web-based interface.

MemExerciser. MemExerciser [24] alleviates episodic memory impairment by recording and later replaying a PwD's passed experiences. The system consists of three parts: mobile sensors for life-logging, the CueChooser application to create a narrative of the PwD's experiences and MemExerciser to replay the narrative. A PwD carries a mobile camera with tree-axis accelerometer (Microsoft SenseCam), an audio recorder and a GPS. The camera and GPS record data in predefined time interval, the audio device records constantly. The recorded information is later preselected by the CueChooser application based on the context meta data. The CG further refines and tags the data to select good memory 
cues for the PwD. The PwD can partly compensate the memory impairment by "recalling" past experiences with the MemExerciser application.

\section{Discussion}

There is an increased focus on early detection and diagnosis of Alzheimer's disease [842]. A recent study commissioned by Alzheimer's Disease International 42 found that early diagnosis and intervention were beneficial to Person with Dementias (PwDs). The motivation for our research is to facilitate the design of assistive applications for people at the early stage of dementia. In this section, we reflect on what we learned about the potential of assistive applications in dementia care to prolong independent living and how People with Dementia (PwDs) and their caregivers (CGs) can be involved in the design process.

\subsection{Assistive Applications to Prolong Independent Living}

The purpose of our work is to help designers to map the needs and requirements of PwDs to the context dimensions activity, identity, location and time [9]. Our literature study shows, that this field is drawing a lot of interest from the research community, which is reflected by several large scale research projects. However, only few applications were tested with the actual target group. This is not surprising, given the following two reasons. First, developing and testing for PwDs is a cumbersome process. The specific disease does not allow a traditional user-centered approach that designs for average users. Designers who collaborate with PwDs face a number of challenges in their effort to understand the requirements and needs of their users 253716. They must develop systems that are tailored to individual users' context to reduce cognitive load, without putting users under pressure, who often provide only vague and sometimes conflicting information 25 37. Second, there is no "user with dementia" archetype a designer can aim for. While PwDs share many characteristics, the advancement of the dementia syndrome is different for each person. Also the PwDs and their CGs's coping strategies will differ from case to case [39]. Designers must therefore not only consider the PwD's cognitive abilities, but also personal and social aspects. This was the main reason our survey focused on projects in which PwDs participated as users. A challenge during our survey was to determine whether a piece of data is context, as defined by Dey and Abowd 9] or not. During the study of assistive software for $\mathrm{PwDs}$, the perspective of context and context-awareness provided us with a first impression of the role of context in the design process.

\subsection{Design Recommendations}

We summarize these findings as a set of basic design recommendations here. The recommendations are distilled from the practices presented by the projects and papers we studied for this survey. We listed good practices that occurred 
in one or more sources, and labeled them with a fitting title. With these design recommendations we want to provide a set of general guidelines and rules that lead to informed design for assistive applications for PwD. Since performing user evaluations with this specific user group is complex and often undesirable, having these guidelines can be of great help to create accessible assistive applications tailored according to the target group.

Represent time explicitly. PwD rely on a concrete notion of time and progress in time to alleviate the impacts of episodic memory impairment. Time is therefore an important non-functional requirement, which is present in all the services we studied. Time is often combined with other context information. E.g. a reminder for medication may also consider the location to present reminders when the $\mathrm{PwD}$ is in the right room. Or an activity assistant may adapt the triggering of prompts depending on the PwD's mood. An appropriate interactive system should schedule events in the interface and display reminders and other information as well as recording time stamps in live logging for later retrieval.

- This recommendation is based on 131647

- This recommendation has been applied by $5[62024 \mid 4445]$

Label temporal events. Temporal impairment can be alleviated by giving a meaning to temporal events. PwDs find it increasingly difficult to make sense of time. This is referred to as semantic time in Table 2. Semantic time is inherently linked to other context dimensions. For example "lunch" is the time when a person eats at home, normally around 12:00h.

- This recommendation is based on 131647.

- This recommendation has been applied by 6 612

Make location data accessible at all times. Use location in the dialog with PwDs to mitigate topological and geographical disorientation. A typical service for the $\mathrm{PwD}$ is a navigation system that guides the person from its current location to a destination. Location also provides tracking capabilities to locate the $\mathrm{PwD}$ and trigger alerts. Semantic location is a location in combination with other context information. For example a shop or a rendezvous point, i.e. a place where two or more people are to meet at a given time. Location also manifests itself in the interface as choices of information of functionalities that are presented to the user. For example a controller removes access to the radio once the $\mathrm{PwD}$ leaves the house.

- This recommendation is based on 41316

- This recommendation has been applied by $5[6 / 2 \mid 45]$

Explicitly and uniquely identify all people, concepts and objects in the user interface. To avoid confusion, label all concepts, people and objects that might be accessed through the user interface. This needs to be done in an unambiguous way to avoid confusing the PwD. E.g. when to contact someone using a contact 
list, people with similar names could cause confusion. In this situation extra information needs to be added to increase the discrepancy between similar items. $\mathrm{PwD}$ tend to misplace objects or be unsure which object to use. Activity assistants and reminders need to know which objects the PwD needs for their tasks. Identity can also be combined with subtypes of activity, such as the status of kitchen appliances.

- This recommendation is based on [13]16]47]

- This recommendation has been applied by [5]6[20[45]

Put the social network central. The first line of help comes from other people: $\mathrm{CG}$, other relatives, medical professionals and friends. The interface can reassure $\mathrm{PwD}$ that there are people they know reachable for help by including a contact list. For example: one presentation of social relationships is an address book that lets a PwD call a contact. An alerting service uses social relationship to send notifications and escalate alerts to multiple contacts is an example for a background service. Additional information about the contacts, such as competencies or availability, can help to route notifications to contacts that are the most likely to help.

- This recommendation is based on [413/3147]

- This recommendation has been applied by [5]6[12]20|45]

Show the current activity at all time. The assistive application should show what the currently planned activity is at all times. This relies on an agenda that is available with the $\mathrm{PwD}$ daily activities. We found "user activity" is, together with time, the most important but also the most demanding to incorporate into the system design. Preferably, the previous activity and next planned activity are included in the user interface design.

- This recommendation is based on 4/16/47/49]

- This recommendation has been applied by [6|12/20|45]

Foster Personal Identity. PwDs and their CGs are very sensitive about how they are perceived by others. Maintaining their personal identity, respect and dignity is vital for PwDs and their CGs. Assistive applications should be appealing to their users and not be perceived as stigmatizing. In addition to functional requirements, the design must therefore consider the individuals' preferences and blend into their environment.

- This recommendation is based on [4|13|16|21|31|34]

- This recommendation has been applied by [6]12/44 45

\section{Conclusion}

The context and context-awareness classification and our design recommendations presented are by no means a substitute for consulting medical professional 
and people affected by dementia. Our aim is to provide a tool to assist a preparatory investigation for the design and development of context-aware applications for Person with Dementia (PwD). The framework informs developers and designers about possible use of context for their applications. Validation with the target group remains the best approach, but given the condition of the test users it is extremely difficult to do actual user trials. Our framework builds upon previous research and development in this domain and makes the previous experiences in building such system more accessible for developers and designers creating a new system. When use cases are defined for a project, our reference framework helps to identify the (1) dimensions of context that need to be taken into account, (2) provide a set of examples of concrete implementations for these dimensions by referring to relevant research projects and (3) helps to uncover important context dimensions that might otherwise easily missed while designing an assistive application for People with Dementia (PwDs).

Acknowledgments. Part of the research described in this article was performed in the context of the IBBT project AToM. This project is cofunded by the IBBT (Interdisciplinary institute for Technology), a research institute founded by the Flemish Government. Companies and organizations involved in the project are Televic Healthcare NV, GS1 Belgium \& Luxembourg vzw, Woonen Zorgcentrum Sint-Jozef vzw, Katholieke Hogeschool Limburg, Ter Linden vzw, Pyxima NV, Bedrijfstechnische Eenheid Broeders Alexianen, with project support of IWT.

\section{References}

1. Aggarwal, N., Vass, A.A., Minardi, H.A., Ward, R., Garfield, C., Cybyk, B.: People with dementia and their relatives: personal experiences of alzheimer's and of the provision of care. J. Psychiatr. Ment Health Nurs 10(2), 187-197 (2003)

2. Association, A.: 2012 alzheimer's disease facts and figures. Alzheimers Dement 8(2), 131-168 (2012)

3. Astell, A., Alm, N., Gowans, G., Ellis, M., Dye, R., Vaughan, P.: Involving older people with dementia and their carers in designing computer based support systems: some methodological considerations. Universal Access in the Information Society 8(1), 49-58 (2009)

4. Brittain, K., Corner, L., Robinson, L., Bond, J.: Ageing in place and technologies of place: the lived experience of people with dementia in changing social, physical and technological environments. Sociology of Health \& Illness 32(2), 272-287 (2010)

5. Chang, Y.J., Tsai, S.K., Wang, T.Y.: A context aware handheld wayfinding system for individuals with cognitive impairments. In: Proceedings of the 10th International ACM SIGACCESS Conference on Computers and Accessibility, Assets 2008, pp. 27-34. ACM, New York (2008)

6. Davies, R.J., Nugent, C.D., Donnelly, M.: Prototyping cognitive prosthetics for people with dementia. In: Jain, L., Wu, X., Mulvenna, M.D., Nugent, C.D. (eds.) Supporting People with Dementia Using Pervasive Health Technologies. Advanced Information and Knowledge Processing, pp. 145-163. Springer, London (2010) 
7. Davies, R.J., Nugent, C.D., Donnelly, M.P., Hettinga, M., Meiland, F.J., Moelaert, F., Mulvenna, M.D., Bengtsson, J.E., Craig, D., Dröes, R.M.: A user driven approach to develop a cognitive prosthetic to address the unmet needs of people with mild dementia. Pervasive and Mobile Computing 5(3), 253-267 (2009); pervasive Health and Wellness Management

8. DeKosky, S.T., Carrillo, M.C., Phelps, C., Knopman, D., Petersen, R.C., Frank, R., Schenk, D., Masterman, D., Siemers, E.R., Cedarbaum, J.M.: Revision of the criteria for alzheimer's disease: A symposium. Alzheimer's and Dementia 7(1), e1-e12 (2011)

9. Dey, A.K., Abowd, G.: Towards a better understanding of context and contextawareness. In: CHI 2000 Workshop on the What, Who, Where, When, and How of Context-awareness, vol. 4, pp. 1-6. Citeseer (2000)

10. Dey, A.K., Abowd, G.D.: CybreMinder: A Context-Aware System for Supporting Reminders. In: Thomas, P., Gellersen, H.-W. (eds.) HUC 2000. LNCS, vol. 1927, pp. 172-186. Springer, Heidelberg (2000)

11. Dey, A.K., Mankoff, J.: Designing mediation for context-aware applications. ACM Trans. Comput.-Hum. Interact. 12, 53-80 (2005)

12. Donnelly, M.P., Nugent, C.D., McClean, S., Scotney, B., Mason, S., Passmore, P., Craig, D.: A mobile multimedia technology to aid those with alzheimer's disease. IEEE Multimedia 17(2), 42-51 (2010)

13. Dröes, R.M., Bentvelzen, S., Meiland, F., Craig, D.: Dementia-related and other factors to be taken into account when developing ict support for people with dementia-lessons from field trials. In: Jain, L., Wu, X., Mulvenna, M.D., Nugent, C.D. (eds.) Supporting People with Dementia Using Pervasive Health Technologies. Advanced Information and Knowledge Processing, pp. 113-127. Springer, London (2010)

14. Du, K., Zhang, D., Zhou, X.-S., Mokhtari, M., Hariz, M., Qin, W.: HYCARE: A Hybrid Context-Aware Reminding Framework for Elders with Mild Dementia. In: Helal, S., Mitra, S., Wong, J., Chang, C.K., Mokhtari, M. (eds.) ICOST 2008. LNCS, vol. 5120, pp. 9-17. Springer, Heidelberg (2008)

15. Gajos, K.Z., Weld, D.S., Wobbrock, J.O.: Automatically generating personalized user interfaces with supple. Artificial Intelligence 174(12-13), 910-950 (2010)

16. Hawkey, K., Inkpen, K.M., Rockwood, K., McAllister, M., Slonim, J.: Requirements gathering with alzheimer's patients and caregivers. In: Proceedings of the 7th International ACM SIGACCESS Conference on Computers and Accessibility, Assets 2005, pp. 142-149. ACM, New York (2005)

17. Hodges, J.R.: Alzheimer's centennial legacy: origins, landmarks and the current status of knowledge concerning cognitive aspects. Brain 129(Pt 11), 2811-2822 (2006)

18. Hoey, J., von Bertoldi, A., Poupart, P., Mihailidis, A.: Assisting persons with dementia during handwashing using a partially observable markov decision process. In: The 5th International Conference on Computer Vision Systems, pp. 1-10 (February 2007)

19. Hoey, J., Plötz, T., Jackson, D., Monk, A., Pham, C., Olivier, P.: Rapid specification and automated generation of prompting systems to assist people with dementia. Pervasive and Mobile Computing (2010) (in Press, Corrected Proof)

20. Hoey, J., Poupart, P., von Bertoldi, A., Craig, T., Boutilier, C., Mihailidis, A.: Automated handwashing assistance for persons with dementia using video and a partially observable markov decision process. Computer Vision and Image Understanding 114(5), 503-519 (2010); special issue on Intelligent Vision Systems 
21. Hughes, J.C., Louw, S.J., Sabat, S.S.R.: Seeing whole. In: Hughes, J.C., Louw, S.J., Sabat, S.S.R. (eds.) Dementia: Mind, Meaning, and the Person, ch. 1, pp. 1-39. Oxford University Press, USA (2006)

22. Kikhia, B., Hallberg, J., Synnes, K., Sani, Z.U.H.: Context-aware life-logging for persons with mild dementia. In: Annual International Conference of the IEEE Engineering in Medicine and Biology Society, EMBC 2009, pp. 6183-6186 (2009)

23. Lauriks, S., Reinersmann, A., Van der Roest, H.G., Meiland, F., Davies, R.J., Moelaert, F., Mulvenna, M.D., Nugent, C.D., Dröes, R.M.: Review of ICT-based services for identified unmet needs in people with dementia. Ageing Research Reviews 6(3), 223-246 (2007)

24. Lee, M.L., Dey, A.K.: Lifelogging memory appliance for people with episodic memory impairment. In: Proceedings of the 10th International Conference on Ubiquitous Computing, UbiComp 2008, pp. 44-53. ACM, New York (2008)

25. Lindsay, S., Brittain, K., Jackson, D., Ladha, C., Ladha, K., Olivier, P.: Empathy, participatory design and people with dementia. In: Proceedings of the 2012 ACM Annual Conference on Human Factors in Computing Systems, pp. 521-530. ACM (2012)

26. Mahmud, N., Vogt, J., Luyten, K., Slegers, K., Van den Bergh, J., Coninx, K.: Dazed and Confused Considered Normal: An Approach to Create Interactive Systems for People with Dementia. In: Bernhaupt, R., Forbrig, P., Gulliksen, J., Lárusdóttir, M. (eds.) HCSE 2010. LNCS, vol. 6409, pp. 119-134. Springer, Heidelberg (2010)

27. Mandell, A.M., Green, R.C.: Alzheimer's Disease, pp. 1-91. Wiley-Blackwell, Oxford (2011)

28. McKeown, J., Clarke, A., Ingleton, C., Repper, J.: Actively involving people with dementia in qualitative research. J. Clin. Nurs. 19(13-14), 1935-1943 (2010)

29. Mihailidis, A., Boger, J., Craig, T., Hoey, J.: The coach prompting system to assist older adults with dementia through handwashing: An efficacy study. BMC Geriatrics 8(1), 28 (2008)

30. Mileo, A., Merico, D., Bisiani, R.: Support for context-aware monitoring in home healthcare. J. Ambient Intell. Smart Environ. 2(1), 49-66 (2010)

31. Monk, A.F.: Simple, social, ethical and beautiful: requirements for uis in the home. In: Proceedings of the Ninth Conference on Australasian user Interface, AUIC 2008, vol. 76, pp. 3-9. Australian Computer Society, Inc., Darlinghurst (2008)

32. Mulvenna, M.D., Moelaert, F., Meiland, F., Sävenstedt, S., Hettinga, M., Craig, D., Bengtsson, J.E., Dröes, R.M., Martin, S.: Designing \& evaluating a cognitive prosthetic for people with mild dementia. In: Caring Technology for the Future: Proceedings of the European Conference on Cognitive Ergonomics, pp. 11-18 (2010)

33. Mulvenna, M.D., Nugent, C.D., Moelaert, F., Craig, D., Dröes, R.M., Bengtsson, J.E.: Supporting people with dementia using pervasive healthcare technologies. In: Jain, L., Wu, X., Mulvenna, M.D., Nugent, C.D. (eds.) Supporting People with Dementia Using Pervasive Health Technologies. Advanced Information and Knowledge Processing, pp. 3-14. Springer, London (2010)

34. Newell, A.F.F., Gregor, P., Morgan, M., Pullin, G., Macaulay, C.: User-sensitive inclusive design. Universal Access in the Information Society 10(3), 235-243 (2011)

35. Newell, A.F., Carmichael, A., Gregor, A., Alm, N., Waller, A.: Information technology for cognitive support. In: Sears, A., Jacko, J.A. (eds.) The Human- Computer Interaction Handbook: Fundamentals, Evolving Technologies, and Emerging Applications, 2nd edn., ch. 41, pp. 811-828. CRC Press (2007) 
36. About alzheimer's disease: Treatment national institute on aging

37. Orpwood, R., Sixsmith, A., Torrington, J., Chadd, J., Gibson, G., Chalfont, G.: Designing technology to support quality of life of people with dementia. Technology and Disability 19(2), 103-112 (2007)

38. Orpwood, R., Sixsmith, A., Torrington, J., Chadd, J., Gibson, M., Chalfont, G.: Designing technology to support quality of life of people with dementia. Technology and Disability 19(2), 103-112 (2007)

39. Perry, J., O'Connor, D.: Preserving personhood (re)membering the spouse with dementia*. Family Relations 51(1), 55-62 (2002)

40. Peterson, C.B., Mitseva, A., Mihovska, A., Prasad, N.R., Prasad, R.: The phenomenological experience of dementia and user interface development. In: 2nd International Symposium on Applied Sciences in Biomedical and Communication Technologies, ISABEL 2009, pp. 1-5 (2009)

41. Phua, C., Foo, V.S.F., Biswas, J., Tolstikov, A., Aung, A.P.W., Maniyeri, J., Huang, W., That, M.H., Xu, D., Chu, A.K.W.: 2-layer erroneous-plan recognition for dementia patients in smart homes. In: 11th International Conference on e-Health Networking, Applications and Services, Healthcom 2009, pp. 21-28 (2009)

42. Prince, M.B., Bryce, R., Ferri, C.: World alzheimer report 2011: The benefits of early diagnosis and intervention. Alzheimer's Disease International 15, 5-65 (2011)

43. Rebenitsch, L., Owen, C.B., Ferrydiansyah, R., Bohil, C., Biocca, F.: An exploration of real-time environmental interventions for care of dementia patients in assistive living. In: Proceedings of the 3rd International Conference on PErvasive Technologies Related to Assistive Environments, PETRA 2010, pp. 34:1-34:8. ACM, New York (2010)

44. Riley, P., Alm, N., Newell, A.: An interactive tool to promote musical creativity in people with dementia. Computers in Human Behavior 25(3), 599-608 (2009)

45. Robinson, L., Brittain, K., Lindsay, S., Jackson, D., Olivier, P.: Keeping In Touch Everyday (KITE) project: developing assistive technologies with people with dementia and their carers to promote independence. International Psychogeriatrics 21(03), 494-502 (2009)

46. Stalker, K., Gilliard, J., Downs, M.G.: Eliciting user perspectives on what works. International Journal of Geriatric Psychiatry 14(2), 120-130 (1999)

47. Wherton, J.P., Monk, A.F.: Technological opportunities for supporting people with dementia who are living at home. International Journal of Human-Computer Studies 66(8), 571-586 (2008)

48. Wherton, J.P., Monk, A.F.: Choosing the right knob. In: Proceedings of the 27th International Conference Extended Abstracts on Human Factors in Computing Systems, CHI 2009, pp. 3631-3636. ACM, New York (2009)

49. Wherton, J.P., Monk, A.F.: Problems people with dementia have with kitchen tasks: The challenge for pervasive computing. Interacting with Computers 22(4), 253-266 (2010); supportive Interaction: Computer Interventions for Mental Health

50. Wimo, A., Prince, M.: World alzheimer report (2010)

51. Wobbrock, J.O., Kane, S.K., Gajos, K.Z., Harada, S., Froehlich, J.: Ability-based design. ACM Transactions on Accessible Computing 3(3), 1-27 (2011) 